\title{
GRP78 mediates radiation resistance of a stem cell-like subpopulation within the MCF-7 breast cancer cell line
}

\author{
BO LI ${ }^{1,2}$, XIAO LIANG CHENG ${ }^{3}$, YE PENG YANG ${ }^{1}$ and ZAI QUAN LI ${ }^{1}$ \\ ${ }^{1}$ Department of Radiation Medicine, Peking University Health Science Center, Beijing 100191; ${ }^{2}$ Department of \\ Nuclear Medicine, Traditional Chinese Medicine Hospital of Henan Province, the Second Affiliated Hospital of \\ Henan Traditional Chinese Medical College, Zhengzhou, Henan 450002; ${ }^{3}$ Department of Pharmacy, \\ First Affiliated Hospital, Medical College of Xi'an Jiaotong University, Xi'an, Shaanxi 710061, P.R. China
}

Received April 24, 2013; Accepted July 21, 2013

DOI: $10.3892 /$ or.2013.2710

\begin{abstract}
Emerging evidence indicates that breast cancerinitiating cells (CICs) are relatively resistant to radiotherapy; however, the critical mechanisms determining breast CIC resistance to radiation remain elusive. In the present study, a subpopulation of cells displaying characteristics generally attributed to stem cells was identified within the breast cancer cell line MCF-7. This subpopulation displays cancer stem cell features characterized by overexpression of embryonic stem cell markers, high tumorigenic potential following transplantation into BALB/c-nu mice, self-renewal capacity and resistance to ionizing radiation (IR). Moreover, glucose-regulated protein 78KD (GRP78), which was found to play a crucial role in stem cell oncogenesis, was also shown to be overexpressed in this subpopulation. GRP78 is required for the cancer stem-like subpopulation cell resistance to IR, as knockdown of this gene augments the effects of IR, while overexpression of GRP78 increases the radiation resistance of the subpopulation to IR. These findings indicate that GRP78 acts as a potential therapeutic target aimed at tumor-generating subsets of breast cancer cells.
\end{abstract}

\section{Introduction}

Radiotherapy remains the standard radiation modality used for the treatment of breast cancer, which is a common malignancy worldwide and carries a high mortality rate (1). Previous studies have shown that radiation improves overall survival from breast cancer in women with early stage and

Correspondence to: Professor Zai Quan Li or Professor Ye Peng Yang, Department of Radiation Medicine, Peking University Health Science Center, 38 Xueyuan Road, Haidian, Beijing 100191, P.R. China

E-mail: yangyepeng@bjmu.edu.cn

E-mail: lizaiquan@bjmu.edu.cn

Key words: breast cancer-initiating cells, side population, glucose-regulated protein $78 \mathrm{KD}$, radiation resistance advanced disease $(2,3)$. Despite the fact that improvements in the management of breast cancer have been made, the clinical application of radiotherapy has improved only marginally, due to the tumor resistance to ionizing radiation (IR). The emerging reasonable explanation for this phenomenon is the existence of a rare subpopulation of cells which are purported cancer stem cells (CSCs) or cancer-initiating cells (CICs) that may be contribute to some cases of resistance to cancer therapy $(4,5)$. Pre-clinical data suggest that breast CSCs/CICs can be enriched after radiation and that breast cancer stem/initiating clonogens are particularly resistant to radiation $(6,7)$. However, the molecular mechanisms that mediate radiation resistance of breast CSCs remain unidentified. Therefore, uncovering key genes which are responsible for maintaining the radiation resistance of breast CSCs is a critical approach for improving the effects of radiotherapy.

Glucose-regulated protein 78KD (GRP78), one of the best-characterized endoplasmic reticulum (ER) chaperones, serves multiple functions in maintaining cellular homeostasis. GRP78 has been implicated as a mediator of tumor proliferation and metastasis, therapeutic resistance and recurrence $(8,9)$. Moreover, recent data indicate that GRP78 plays a crucial role in stem cell biology (10). For instance, GRP78 is required for the survival of embryonic stem cell precursors and is also highly expressed in hematopoietic stem cells (11). Additionally, GRP78 has been reported to be highly elevated in breast disseminated tumor cells, which shared similar biological properties of CICs (12). In agreement, differential systemic analysis revealed elevated GRP78 expression in head and neck CICs (13). However, the role of GRP78 in breast CICs has yet to be determined. Based on these findings, it is worth investigating the role of GRP78 in breast CICs if GRP78 is preferentially overexpressed in CICs.

In order to test this hypothesis, we first identified breast CICs from MCF-7 cell lines by utilizing flow cytometry based cell-sorting base on ABCG2 efflux pump-mediated Hoechst 33342 dye exclusion, which enables the isolation of a rare stem-like side population (SP) cells (14-17). This method allows estimating the GRP78 expression and determining whether isolated SP fraction which harbors the 
cancer stem cell-like properties is involved in resistance to radiation. This, in turn, could provide an explanation as to why tumors have some degree of intrinsic resistance to radiotherapy.

\section{Materials and methods}

Cell culture and reagents. The MCF-7 breast cancer cell line was purchased from the American Type Culture Collection (Manassas, VA, USA) and cultured in Roswell Park Memorial Institute (RPMI)-1640 culture medium (Macgene, Beijing, China) supplemented with $10 \%(\mathrm{v} / \mathrm{v})$ fetal bovine serum (FBS; Hyclone, Utah, USA) at $37^{\circ} \mathrm{C}$ in a humidified atmosphere containing $5 \% \mathrm{CO}_{2}$. Hoechst 33342 , epidermal growth factor (EGF), human recombinant basic fibroblast growth factorbasic (bFGF), B27 supplement and Lipofectamine ${ }^{\circledR} 2000$ were purchased from Invitrogen (Carlsbad, CA, USA). Verapamil was obtained from Sigma (St. Louis, MO, USA) and dissolved in distilled deionized water $\left(\mathrm{ddH}_{2} \mathrm{O}\right)$. Serum-free Opti-MEM ${ }^{\circledR} \mathrm{I}$ and DMEM/F12 medium were purchased from Gibco (Grand Island, NY, USA).

Analysis and isolation of $S P$ and non-SP cell fractions from MCF-7. The SP analysis was performed based on the method reported by Goodell et al (14) with slight modifications. MCF-7 cells were digested with $0.25 \%$ trypsin (Sigma), washed twice with PBS, resuspended in pre-warmed RPMI-1640 culture (supplemented with $2 \%$ FBS) at a density of $1 \times 10^{6}$ cells $/ \mathrm{ml}$. Then, the cells were pre-incubated with or without $50 \mu \mathrm{M}$ verapamil for $30 \mathrm{~min}$ at $37^{\circ} \mathrm{C}$ before adding the Hoechst 33342 dye at a final concentration of $5 \mu \mathrm{g} / \mathrm{ml}$. The mixture was incubated in the dark at $37^{\circ} \mathrm{C}$ for 90 min with interval mixing. Following incubation, the cells were washed twice with ice-cold PBS, and the cells were then filtered through a $40 \mu \mathrm{m}$ nylon mesh to obtain single cell suspension and kept at $4^{\circ} \mathrm{C}$ in the dark. Cell analysis and purification were performed using FACS (FACSAria II; Becton-Dickinson, CA, USA). Hoechst 33342 was excited with UV light at $355 \mathrm{~nm}$ and fluorescence emission was measured with 450/BP50 (Hoechst blue) and a 660/BP50 (Hoechst red) optical filters. At the end of sorting, both collected SP and non-SP cells were reanalyzed to evaluate sorting purity and to conduct further experiments. To minimize the non-specific effects of the Hoechst dye on the sorted cells, we cultured both SP and non-SP cells for $24 \mathrm{~h}$ to remove dead cells and then performed all experiments described below.

Long-term differentiation of SP and non-SP cells. Sorted SP and non-SP cells were cultured in RPMI-1640 (supplemented with $10 \%$ FBS) for 28 days. Then the cultured SP and non-SP cells were stained with Hoechst 33342 and analyzed by FACS to determine the differentiation ability of the two subpopulations.

Sphere formation and clone formation assay. Sorted SP and non-SP cells were cultured in tumor sphere medium consisting of serum-free DMEM/F12 medium, B27 supplement, $20 \mathrm{ng} / \mathrm{ml}$ EGF and $10 \mathrm{ng} / \mathrm{ml} \mathrm{bFGF}$. Cells were plated at a density of $5 \times 10^{3}$ cells/well in ultra-low-attachment 6-well plate triplicates and the medium was changed every other day. After 10 days in culture, colonies that contained $>20$ cells were counted.
For clone formation assay, sorted SP and non-SP cells were plated in triplicate at 600 cells on each $25-\mathrm{cm}^{2}$ flasks and cultured with RPMI-1640 (supplemented with 10\% FBS) for 10 days. Then, cells were fixed and stained with $0.5 \%$ crystal violet. Colonies containing $>50$ cells were manually counted. The clone formation efficiency was the ratio of the clone number to the planted cell number.

Tumorigenicity assay. Numbers of sorted SP and non-SP cells $\left(1 \times 10^{5}\right)$ suspended in $200 \mu 1$ PBS were injected subcutaneously in the flank region of 5-week-old female BALB/c nude mice obtained from the Institute of Laboratory Animal Science of Peking University Health Science Center. The mice were monitored weekly and euthanized 4 weeks after transplantation to assess tumor formation. Tumors were measured using a vernier caliper, weighed and photographed. Tumor volume (TV) was calculated using the following formula: $\mathrm{TV}\left(\mathrm{mm}^{3}\right)=\left(\right.$ length $\left.\mathrm{x} \mathrm{width}^{2}\right) / 2$. A portion of the subcutaneous tumor tissue was collected, fixed in $10 \%$ formaldehyde and embedded in paraffin for hematoxylin and eosin (H\&E) staining to assess tumor pathology. All animal practices were in accordance with the guidelines of Peking University Health Science Center for the use of laboratory animals.

Radiation and clonogenic assay. Subsequently, 600-8,000 cells were plated on $25-\mathrm{cm}^{2}$ flasks in triplicate for each experiment. Twelve hours later, the cells irradiated at room temperature with a ${ }^{60} \mathrm{Co}$ laboratory irradiator (Beijing Normal University, Beijing) at a dose rate of $1 \mathrm{~Gy} / \mathrm{min}$ for the time required to generate a dose curve of $0,0.5,1,2,4$, 6 and $8 \mathrm{~Gy}$. The control was sham irradiated. Following the irradiation, the cells were incubated for an additional 9 days, and the cells were fixed with $100 \%$ carbinol and stained with $0.5 \%$ crystal violet. Only colonies containing $>50$ cells were manually counted. The surviving fraction was calculated as follows: plating efficiency $(\mathrm{PE})=($ colony number/inoculating cell number) $\mathrm{x} 100 \% . \mathrm{SF}=\mathrm{PE}$ (tested group)/PE $(0 \mathrm{~Gy}$ group) $\mathrm{x} 100 \%$. The cell-survival was calculated according to the single-hit multi-target formula: $\mathrm{SF}=1-\left(1-\mathrm{e}^{-\mathrm{D} / \mathrm{D} 0}\right)^{\mathrm{N}}(18)$. The radiobiological parameters of cellular radiosensitivity $\left(D_{0}\right.$, mean lethal dose), the capacity for sublethal damage repair $\left(D_{q}\right.$, quasi-threshold dose) and the extrapolation number $(\mathrm{N})$ were calculated. Then, those values were used to calculate the SF after irradiation at a dose of $2 \mathrm{~Gy}$ (SF2) and the sensitization enhancement ratio (SER).

$R N A$ extraction and quantitative real-time PCR. Total RNA was extracted from newly sorted SP and non-SP cells using Takara RNAiso Plus (Dalian, China) and reverse transcriptions were performed according to the protocol supplied by the manufacturer (Takara, Japan).

Real-time PCR was performed using SYBR-Green I master mix kit (Takara) on a Bio-Rad IQ5 Real-time-PCR Reaction System (Bio-Rad Laboratories, Inc., CA, USA). The relative amounts of mRNA were calculated from the values of comparative threshold cycle by using GAPDH as control (primers are depicted in Table I). The reaction was carried out with the following cycling conditions: $95^{\circ} \mathrm{C}$ for $2 \mathrm{~min}$ followed by 45 cycles of amplification (denaturation at $95^{\circ} \mathrm{C}$ for $15 \mathrm{sec}$, annealing at $58^{\circ} \mathrm{C}$ for $20 \mathrm{sec}$ and extension at $72^{\circ} \mathrm{C}$ for $30 \mathrm{sec}$ ). 
Table I. Primer sequences used for the real-time PCR.

\begin{tabular}{|c|c|c|c|}
\hline Gene & Primer & Product size (bp) & Temperature $\left({ }^{\circ} \mathrm{C}\right)$ \\
\hline $\begin{array}{l}\text { ABCG2 } \\
\left(\mathrm{NM} \_004827\right)\end{array}$ & $\begin{array}{l}\text { S: 5'-CATGTACTGGCGAAGAATATTTGGT-3' } \\
\text { A: 5'-CACGTGATTCTTCCACAAGCC-3' }\end{array}$ & 74 & $\begin{array}{l}65.2 \\
64.3\end{array}$ \\
\hline $\begin{array}{l}\text { Bmi1 } \\
\left(\mathrm{NM}_{-} 005180\right)\end{array}$ & $\begin{array}{l}\text { S: 5'-AAATGCTGGAGAACTGGAAAG-3' } \\
\text { A: 5'-CTGTGGATGAGGAGACTGC-3' }\end{array}$ & 124 & $\begin{array}{l}60.9 \\
61.1\end{array}$ \\
\hline $\begin{array}{l}\text { Nanog } \\
(\text { NM_024865) }\end{array}$ & $\begin{array}{l}\text { S: 5'-ATTCAGGACAGCCCTGATTCTTC-3' } \\
\text { A: 5'-TTTTTGCGACACTCTTCTCTGC-3' }\end{array}$ & 76 & $\begin{array}{l}65.5 \\
64.5\end{array}$ \\
\hline $\begin{array}{l}\text { Sox } 2 \\
(\mathrm{NM}+003106)\end{array}$ & $\begin{array}{l}\text { S: 5'-CGAGTGGAAACTTTTGTCGGA-3' } \\
\text { A: 5'-TGTGCAGCGCTCGCAG-3' }\end{array}$ & 74 & $\begin{array}{l}63.3 \\
63.4\end{array}$ \\
\hline $\begin{array}{l}\text { Oct4 } \\
(\mathrm{NM}+002701)\end{array}$ & $\begin{array}{l}\text { S: 5'-GTGGAGAGCAACTCCGATG-3' } \\
\text { A: 5'-TGCTCCAGCTTCTCCTTCTC-3' }\end{array}$ & 86 & $\begin{array}{l}61.8 \\
63.4\end{array}$ \\
\hline $\begin{array}{l}\text { GRP78 } \\
(\text { NM_005347) }\end{array}$ & $\begin{array}{l}\text { S: 5'-CACGCCGTCCTATGTCGC-3' } \\
\text { A: 5'-AAATGTCTTTGTTTGCCCACC-3' }\end{array}$ & 238 & $\begin{array}{c}60 \\
55.9\end{array}$ \\
\hline $\begin{array}{l}\text { GAPDH } \\
\text { (NM_002046) }\end{array}$ & $\begin{array}{l}\text { S: 5'-AATTGAGCCCGCAGCCTCCC-3' } \\
\text { A: 5'-CCAGGCGCCCAATACGACCA-3' }\end{array}$ & 153 & $\begin{array}{l}69.7 \\
69.3\end{array}$ \\
\hline
\end{tabular}

$\mathrm{S}$, sense; A, antisense.

Transient overexpression and silencing of GRP78 in SP cells. To overexpress and silence the GRP78 in SP cells, the plasmid (pcDNA3.1 /hGRP78, a gift from Dr RC Austin, McMaster University, Ontario, Canada; pSuper /GRP78 RNAi, designed by our laboratory) which can overexpress and silence the GRP78 in mammalian cells was introduced by transfection. For transient transfection, SP cells were cultured in 6-well plates and transfected at $80 \%$ confluence with Lipofectamine ${ }^{\circledR}$ 2000 according to the manufacturer's instructions. After transfection, the cells were left for another $36 \mathrm{~h}$ before they were harvested by trypsinization and resuspended for clonogenic experiments. The siRNA sequences for human GRP78 are: sense 5'-GATCCCCGATCACAATCACCAATGACTTCAA GAGAGTCATTGGTGATTGTGATCTTTTTA-3' and antisense 5'-AGCTTAAAAAGATCACAATCACCAATGACTC TCTTGAAGTCATTGGTGATTGTGATCGTG-3'.

Statistical analysis. Statistical analysis was performed with Sigmaplot 10.0 software. Statistical differences between SP and non-SP cells were analyzed using the Student's t-test. Data are presented as the means \pm SEM. P-values $<0.05$ were considered to indicate statistically significant differences.

\section{Results}

Isolation of SP and non-SP cell fractions from MCF-7 cell line. We first attempted to isolate a side-population within $\mathrm{MCF}-7$ breast cancer cell lines. Following trypsinization and staining with fluorescent dye Hoechst 33342, the cells were analyzed by flow cytometry; the P2 gate showed that the SP cells were Hoechst 33342 negative/low and the P3 gate indicated the non-SP cells that were Hoechst 33342 positive (Fig. 1A). MCF-7 cells presented a distinct SP, accounting for $3.3 \%$ of the whole population. The percentage of SP cells diminished to $\sim 0.0 \%$ of the total cells when pretreated with verapamil
(Fig. 1B), confirming that SP cells extrude Hoechst 33342 dye actively via a verapamil-sensitive ABC transporter. Then the SP (P2) and non-SP (P3) cells were sorted separately and applied for further experiments; the purity of SP and non-SP cells was 94.3 and $99.7 \%$, respectively (Fig. 1C and D).

SP cells show high tumorigenicity and clonogenic capacity. High tumorigenic potential is considered a hallmark of CICs (19). We first performed tumorigenicity assay to compare the tumorigenic potential of SP and non-SP cells, respectively. Both $1 \times 10^{5}$ numbers of SP and non-SP cells were injected into the flank region of nude mice subcutaneously $(n=3)$. Four weeks after inoculation, both SP and non-SP cells were able to produce tumors as shown in Fig. 2A. Then, the mice were euthanized and the tumors were measured with a vernier caliper. The results showed that SP cells formed a tumor with a mean volume of $1043.12 \pm 163.65 \mathrm{~mm}^{3}$ while non-SP cells formed tumor with $204.04 \pm 144.86 \mathrm{~mm}^{3}$ mean volume (Fig. 2B). H\&E staining results confirmed that the tumors formed by SP and non-SP cells were typical adenomatous carcinoma (Fig. 2C). $\mathrm{H} \& \mathrm{E}$ staining of tumors grown in mice after injection of SP cells showed the presence of malignant cells, with large nuclei and prominent nucleoli; some cells showed a dark basophilic cytoplasm. Moreover, cells were in a chaotic arrangement with necrosis (Fig. 2C).

Additionally, the clone formation assay showed that the mean clone formation efficiency was $77.56 \pm 3.67$ and $26.39 \pm 3.25 \%$ in SP and non-SP cells, respectively (Fig. 2D). In vitro clonogenic potential indicated the efficiency of SP and non-SP cells to form a tumor, which is consistent with the in vivo tumor transplant results.

SP cells harbor long-differentiation ability. SP cells and non-SP cells were cultured for 4 weeks in normal RPMI-1640 medium, stained again with Hoechst 33342 and analyzed using 

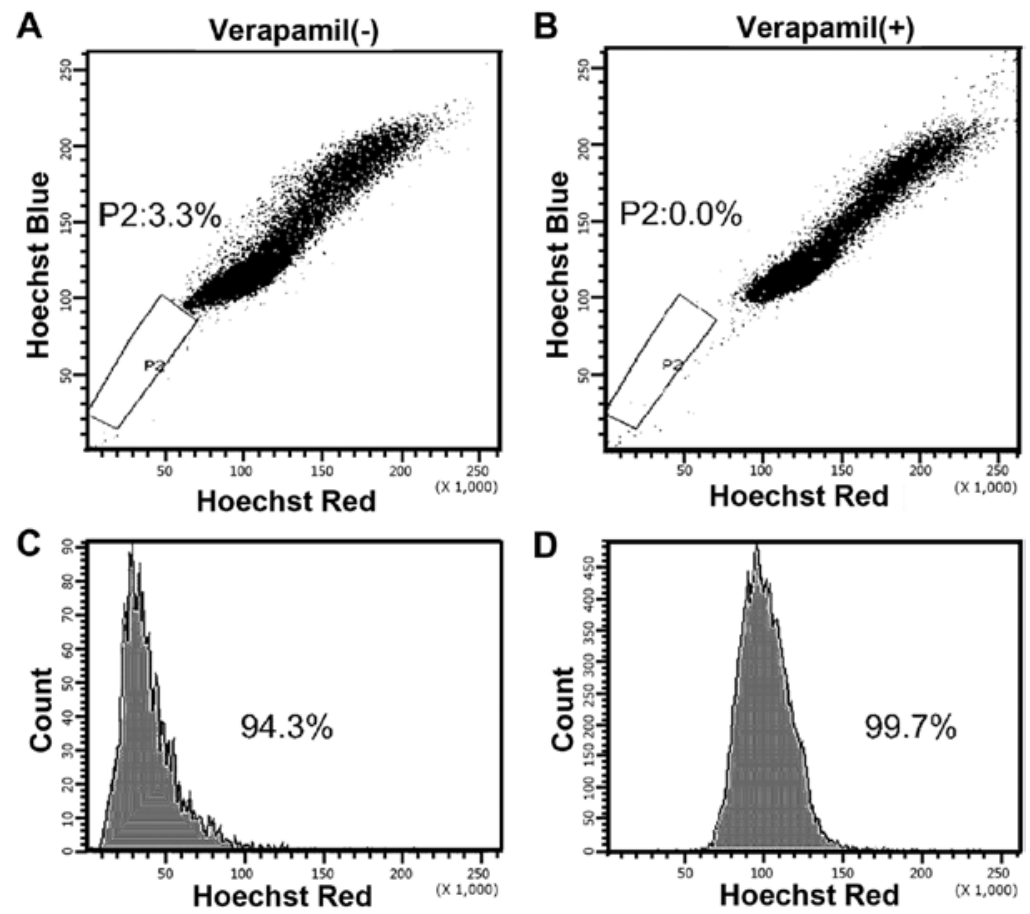

Figure 1. Side population (SP) assay and sorting results. (A) MCF-7 cells stained with Hoechst 33342 and subsequently analyzed by flow cytometry as described in Materials and methods. The SP cells (P2 gate) and non-SP (P3 gate) cells were gated and collected for subsequent research. (B) The SP fraction was dropped to $0.0 \%$ when the cells were pre-incubated with verapamil to block the ATP transporter. (C and D) The sorting purity of the freshly sorted SP and non-SP cells was 94.3 and $99.7 \%$, respectively.

\section{A}

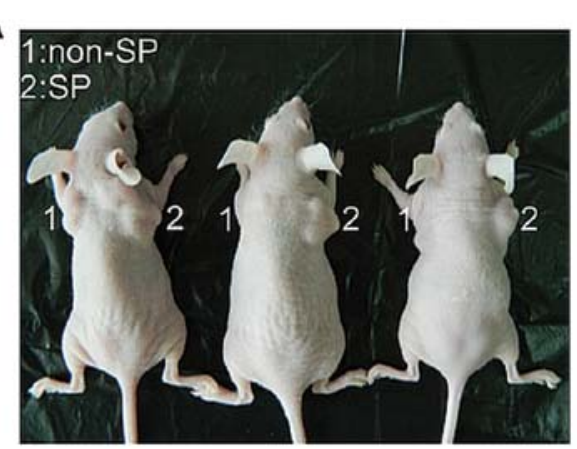

C

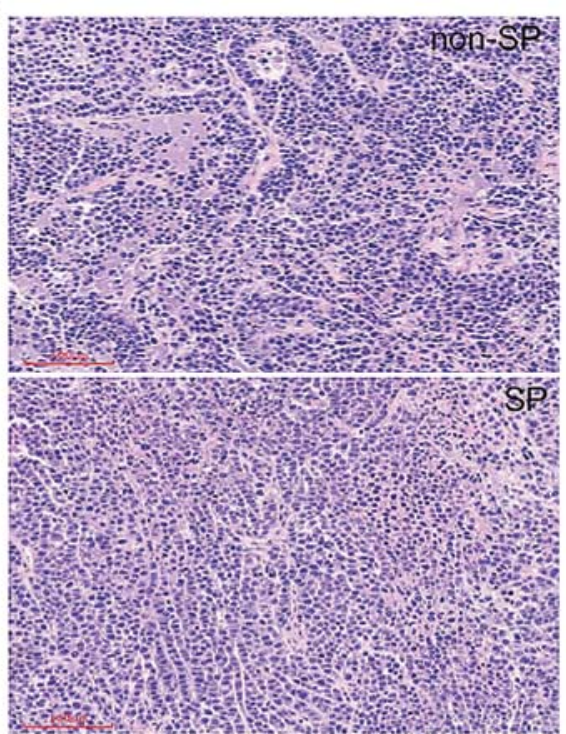

B

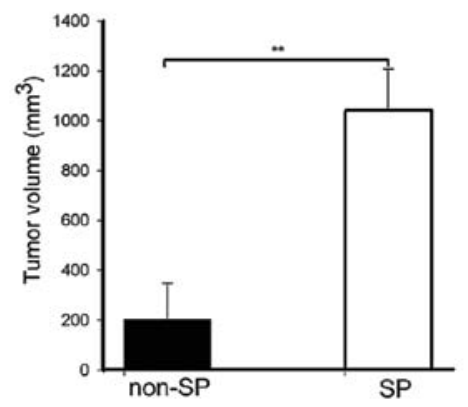

D

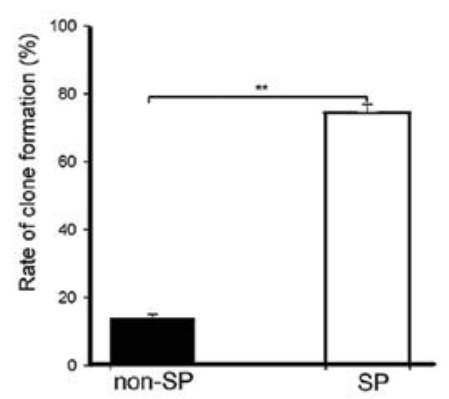

Figure 2. The side population (SP) cells show high tumorigenicity and clonogenic capacity. (A) Representative subcutaneous tumors due to the injection of $1 \times 10^{5}$ non-SP and SP cells. (B) Tumor volume of non-SP and SP cells was measured. Error bars correspond to SD $\left({ }^{* *} \mathrm{P}<0.01\right)$. (C) H\&E staining of tumors derived from non-SP and SP cells (x10 objective).(D) The SP cells display higher clone-forming capacity (>50 cells/clone) than non-SP cells. Error bars correspond to $\mathrm{SD}\left({ }^{* *} \mathrm{P}<0.01\right)$. 

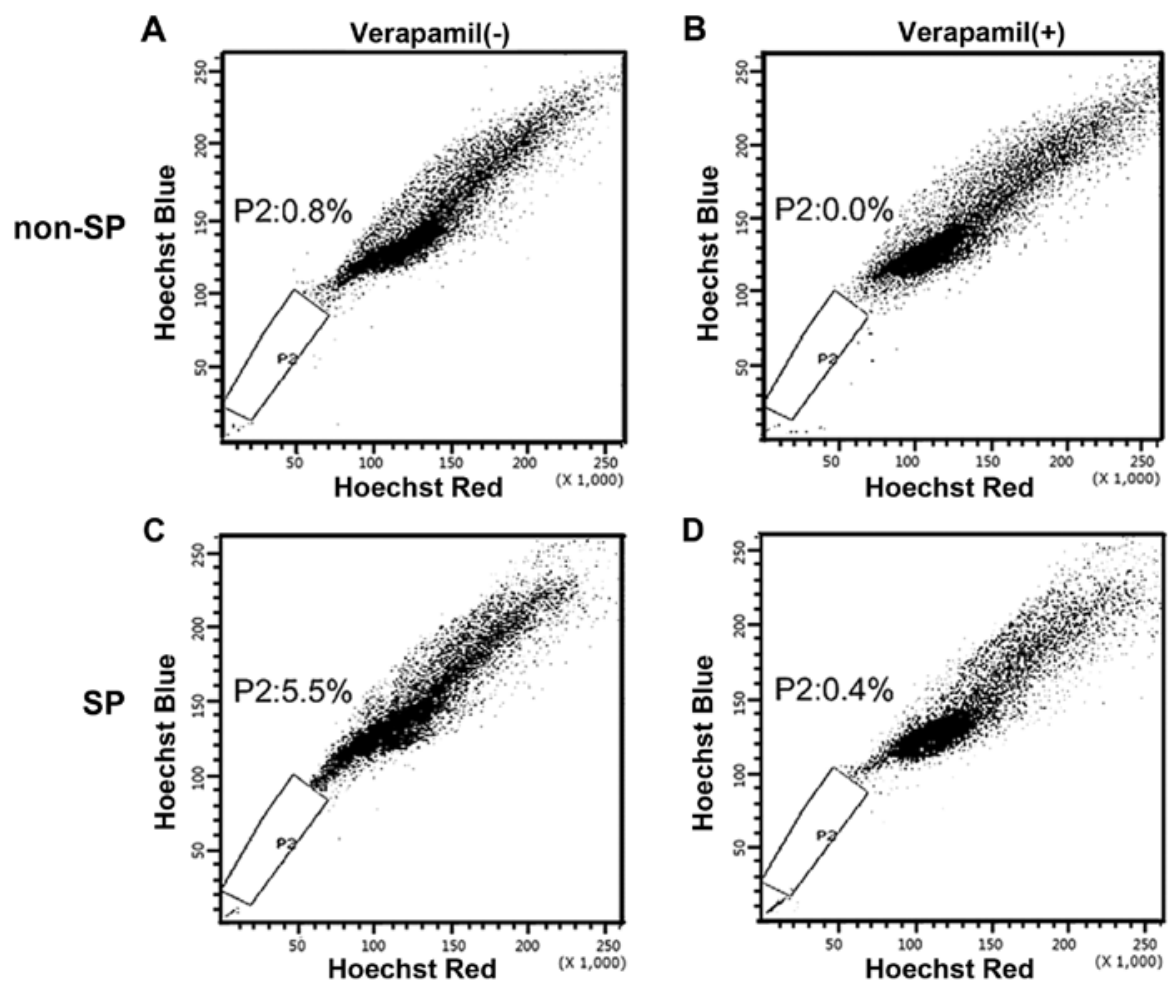

Figure 3. The side population (SP) cells can differentiate into non-SP cells. (A-D) Non-SP and SP cells were reanalyzed by flow cytometry after culturing for 4 weeks in normal RPMI-1640 medium. SP and non-SP subpopulations were obtained again from the former SP cells. By contrast, non-SP cells produced only non-SP fraction.

the cell sorter. As shown in Fig. 3, SP and non-SP fractions were obtained again from the former SP subpopulation after being cultured for 4 weeks (Fig. 3C). By contrast, SP fraction could not be obtained from the former non-SP population (Fig. 3D). This indicated that SP cells may undergo asymmetrical division to generate heterogeneous phenotypes of low-tumorigenic cells, such as non-SP cells that form the bulk of the tumor.

SP cells display higher stemness gene expression, self-renewal ability and resistance to $I R$. Sphere formation has been well described as a typical characteristic of CICs that reflects the potential for self-renewal (20). We evaluated the ability of SP and non-SP cells to generate spherical colonies in an ultra-low attachment and serum-starved culture system. After 10 days of culture, spherical colonies were counted. As shown in Fig. 4A, SP cells displayed much higher tumor sphere-forming ability than non-SP cells; the sphere formation efficiency was $10.17 \pm 2.33 \%$ for the SP cells vs. $2.33 \pm 0.25 \%$ for the non-SP cells (Fig. 4B).

To further confirm the stem phenotype of the SP cells, the expression of embryonic stem cell (ES) marker genes were also investigated, as CICs are considered to share similar characteristics with normal stem cells (21). As shown in Fig. 4C, the q-PCR analysis indicated that the mRNA expression of stemness genes such as Oct4, Nanog, Sox 2 and Bmi1 in SP cells was significantly higher than non-SP cells. Furthermore, the results also showed that the mRNA levels of ABCG2 in SP cells were more highly expressed as compared to non-SP cells, according to the sorting phenotype of Hoechst 33342 exclusion.

Current data demonstrate that breast CICs can be enriched after radiation and $\mathrm{CSCs} / \mathrm{CICs}$ are particularly more resistant to radiotherapy $(6,7,22)$. To compare the SP and non-SP cell
Table II. The survival fraction of 2 Gy-irradiated cells in different treatment.

\begin{tabular}{lcc}
\hline Treatment & $\begin{array}{c}\text { SF 2 Gy } \\
(\text { mean } \pm \text { SEM })^{\mathrm{a}}\end{array}$ & P-value $^{\mathrm{b}}$ \\
\hline non-SP & $0.570 \pm 0.046$ & 0.002 \\
SP & $0.774 \pm 0.022$ & \\
pcDNA3.1(+) & $0.675 \pm 0.033$ & 0.004 \\
pcDNA3.1(+)/hGRP78 & $0.791 \pm 0.012$ & \\
pSuper & $0.800 \pm 0.059$ & 0.010 \\
pSuper/GRP78 RNAi & $0.596 \pm 0.049$ & \\
\hline
\end{tabular}

${ }^{\mathrm{a}} \mathrm{n}=3$. ${ }^{\mathrm{b} P} \mathrm{P}$-value was derived from independent-sample t-test. SF $2 \mathrm{~Gy}$, the survival fraction after 2 Gy irradiation; SP, side population.

response to radiation-induced cytotoxicity, clonogenic assays were performed. The dose-dependent survival curves of SP and non-SP cells are presented in Fig. 4D, the SP cells exhibited more resistance than the non-SP cells. Accordingly, SP cells had larger survival fraction values at 2 Gy irradiation than non-SP cells (Table II). By application of the single-hit multi-target model, the values of $\mathrm{D}_{0}, \mathrm{~N}$ and $\mathrm{D}_{\mathrm{q}}$ of SP and non-SP cells were analyzed. As shown in Table III, SP cells display significantly larger values of $\mathrm{D}_{\mathrm{q}}$ than non-SP cells, indicating that enhanced repair of sublethal damage may contribute to higher surviving fraction after irradiation in SP cells.

GRP78 has been hypothesized to be a key regulator of the therapeutic resistance properties of cancer stem-like cells. 
Table III. Radiobiological parameters from different treatment.

\begin{tabular}{lcccc}
\hline Treatment & \multicolumn{1}{c}{$\mathrm{D}_{0}$} & $\mathrm{~N}$ & $\mathrm{D}_{\mathrm{q}}$ & $\mathrm{SERD}_{\mathrm{q}}$ \\
\hline non-SP & $2.210 \pm 0.564$ & $1.914 \pm 0.895$ & $1.082 \pm 0.437$ & $0.777 \pm 0.408$ \\
SP & $2.111 \pm 0.177$ & $2.811 \pm 0.607$ & $2.127 \pm 0.124$ & \\
pcDNA3.1(+) & $2.330 \pm 0.046$ & $1.960 \pm 0.181$ & $1.560 \pm 0.063$ & $0.823 \pm 0.058$ \\
pcDNA3.1(+)/hGRP78 & $2.178 \pm 0.153$ & $3.310 \pm 0.735$ & $2.552 \pm 0.108$ & \\
pSuper & $2.494 \pm 0.131$ & $2.645 \pm 0.414$ & $2.390 \pm 0.088$ & $1.348 \pm 0.094$ \\
pSuper/GRP78 RNAi & $2.349 \pm 0.139$ & $1.737 \pm 0.204$ & $1.277 \pm 0.110$ & \\
\hline
\end{tabular}

Values are mean $\pm \operatorname{SEM}(n=3) . D_{0}$, the mean lethal dose; $N$, the extrapolation number, a parameter to measure the width of shoulder of the survival curve; $\mathrm{D}_{\mathrm{q}}$, quasi-threshold dose; $\mathrm{SER}$, sensitization enhancement ratio.
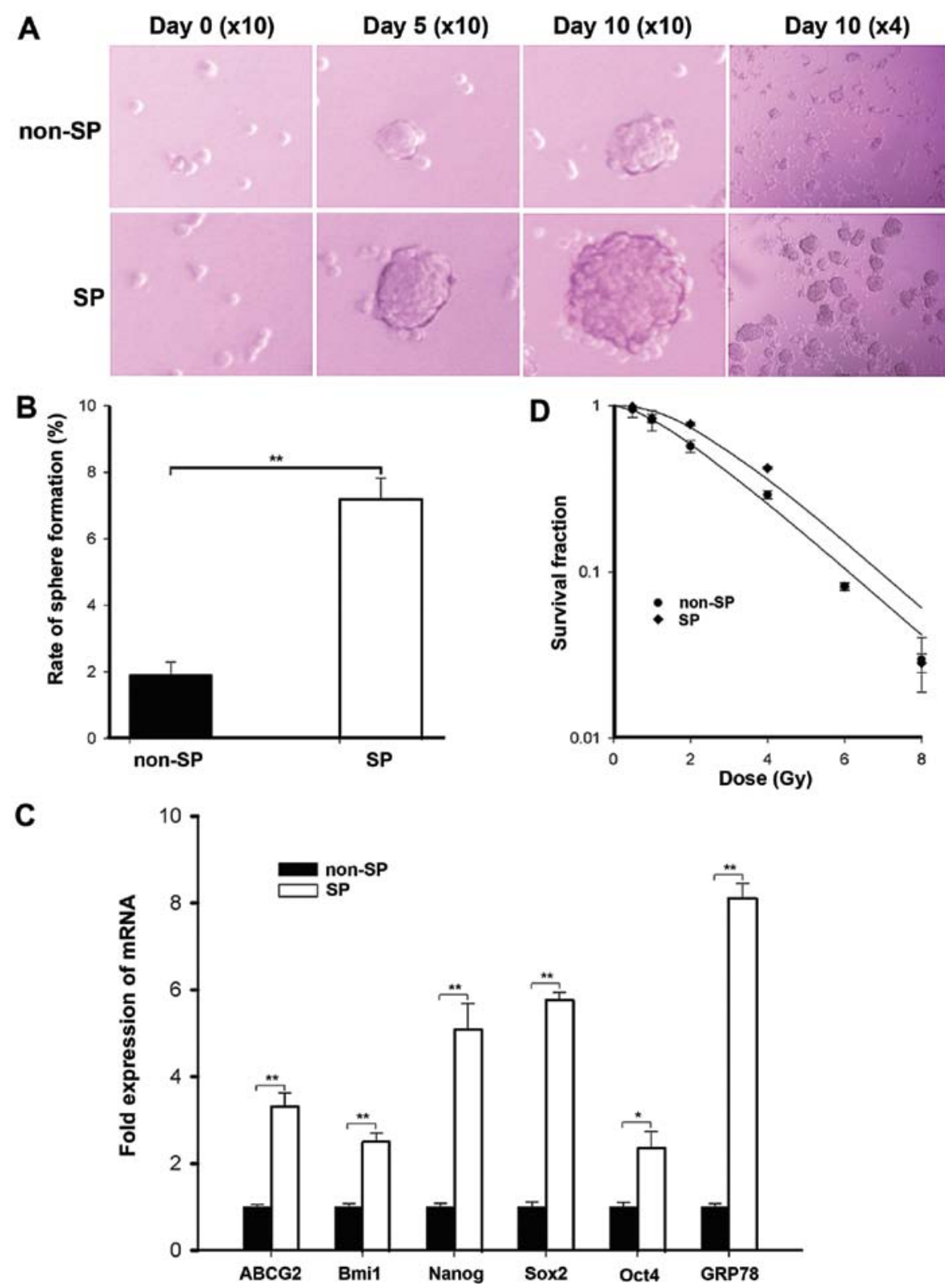

Figure 4. The side population (SP) cells display higher sphere formation ability, stemness gene expression and resistance to ionizing radiation (IR). (A) Sorted non-SP and SP cells were plated in ultra-low-attachment 6-well culture dishes as previously described, and the formation of mammospheres was photographed (x4 objective) on the indicated day. (B) SP cells had significantly enhanced sphere-forming ability compared with the non-SP cells. Error bars correspond to $\mathrm{SD}\left({ }^{* *} \mathrm{P}<0.01\right)$. (C) q-PCR analysis demonstrated the mRNA levels of ABCG2, Bmi1, Nanog, Oct4, Sox2 and glucose-regulated protein 78KD (GRP78) were higher in SP than in non-SP cells. Error bars correspond to SD $\left({ }^{*} \mathrm{P}<0.05,{ }^{* *} \mathrm{P}<0.01\right)$. (D) Clonogenic survival assay of non-SP and SP cells after irradiation was performed as described in Materials and methods. The survival curves represent the data as fit by single-hit multi-targets model. SP cells exhibited increasing resistance to IR compared with non-SP cells. 

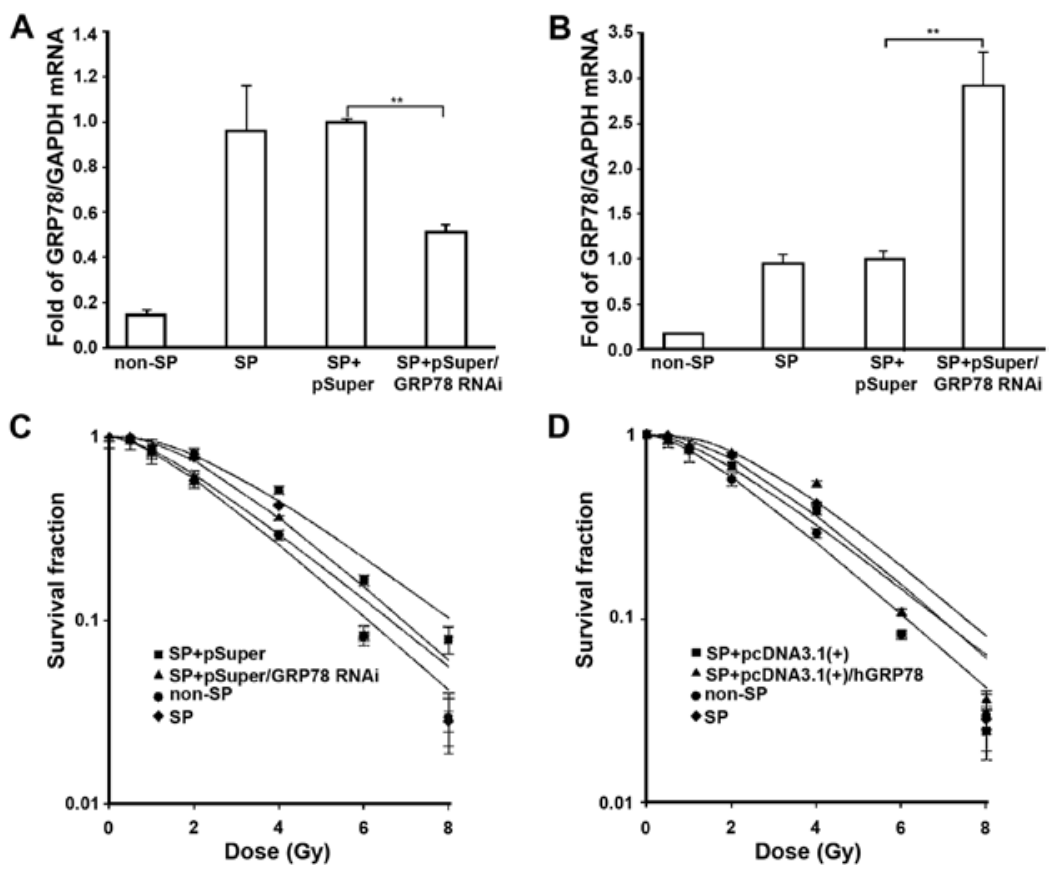

Figure 5. GRP78 is necessary for maintaining the radiation resistance properties of SP cells. (A and B) Downregulation and upregulation of GRP78 in SP cells mediated by pSuper/GRP78 RNAi and pcDNA3.1(+)/hGRP78 vector, respectively. The mRNA expression of GRP78 was then validated by real-time PCR. Error bars correspond to SD ( $\left.{ }^{* *} \mathrm{P}<0.01\right)$. (C and D) Clonogenic survival assay was performed as described in Materials and methods. (C) Silencing the GRP78 expression in SP cells exhibited increasing sensitivities to radiation, while (D) overexpression of GRP78 significantly reduced the effects of ionizing radiation.

We then examined the GRP78 expression in SP cells. q-PCR analysis demonstrated that the expression level of GRP78, which is required for survival of embryonic stem cell precursors, was highly expressed in SP cells (Fig. 4C).

GRP78 is necessary for maintaining the radiation resistance properties of SP cells. To evaluate the correlation between GRP78 expression profile and radiation resistance, the GRP78 gene was silenced using a small interfering RNA expressed in a pSuper vector. The SP cells were transfected with pSuper and pSuper/GRP78 RNAi vector. After 36 h transfection, q-PCR analysis confirmed that the expression of GRP78 was markedly suppressed in pSuper/GRP78 RNAi vector-transfected SP cells (Fig. 5A). Then, the transfected cells were harvested by trypsinization and resuspended for clonogenic experiments. As shown in dose-dependent survival curves (Fig. 5C), the IR effect on SP cells treated with pSuper/GRP78 RNAi vector was significantly stronger as compared to SP cells treated with a scrambled control. These data demonstrated that the silencing of GRP78 significantly enhanced the sensitivity of SP cells to IR.

In order to further verify the role of GRP78 in the resistance of SP cells to irradiation, we performed the gain-of-function approach by transfection with pcDNA3.1(+)/ hGRP78 plasmids transient overexpressing GRP78 into SP cells. Total mRNA from SP cells with transfection of GRP78-expressing plasmids displayed elevated expression of GRP78 (Fig. 5B). Then, exposed to IR, the results indicated that GRP78 overexpression resulted in increased radiation resistance in SP cells (Fig. 5D). As shown in Tables II and III, the survival fraction of 2 Gy and radiobiological parameters demonstrated that knockdown of the GRP78 gene increases the effects of IR, while overexpression of GRP78 decreases the radiation-induced cytotoxicity to
SP cells. Collectively, our data provide evidence that GRP78 upregulation in SP cells mediates the resistance to IR.

\section{Discussion}

Despite advances in the detection and treatment of breast cancer, mortality from this disease remains high as current therapies are limited by the emergence of therapy resistance $(23,24)$. Several reports support the existence of a subset of cells bearing stem cell characteristics within breast tumors $(20,25)$, giving rise to the possibility that tumor therapy resistance is mainly due to the CSC-resistance to antitumor treatment $(26,27)$. Phillips et al (6) and Lagadec et al (7) reported that a population of $\mathrm{CD} 24^{-/ \text {low }} / \mathrm{CD} 44^{+}$cells, which regards the breast progenitor cells were resistant to radiation and the population of CICs increased during the course of fractionated radiation. This provided strong support for the hypothesis that CSCs are responsible for the resistance to cancer treatment.

In order to investigate the role of CICs and the molecular characteristics in radiation resistance, the first key step is to identify and isolate CICs. As previously described, reports clearly support that a functionally distinct subpopulation of CICs can be isolated from breast cancer using either prospective surface marker-based FACS analysis or SP cell sorting $(15-17,20)$. The isolation of SP cells is based on the technique initially described by Goodell et al (14). SP assay has emerged as a promising method for identifying cancer stem-like cell and progenitor populations in different types of cancer $(16,17,28,29)$. In the present study, we isolated SP cells from human MCF-7 cell lines, helping to further characterize the biological properties of this cell type.

Compared with the bulk of non-SP cancer cells, SP cells have been shown to display increased ability to form tumor spheres 
and had a high clonogenic efficiency and tumorigenicity when transplanted into immunocompromised mice (Figs. 2 and 4). The SP cells also displayed higher ES cell marker expression (e.g., Bmi1, Nanog, Oct4 and Sox2) (Fig. 4C) and self-renewal capacity. SP cells can differentiate into the bulk of non-SP cells (Fig. 3). Our result shows that the SP cells are more resistant to radiation than non-SP cells, which is consistent with findings of other reports that CICs are more resistant to IR. These results provide direct evidence that the SP cells we sorted bear some of the phenotypic characteristics of CICs.

Although the putative breast CICs are considered to be mediators of resistance to current therapies, the underlying molecular mechanism determining the radiation resistance remains elusive. Currently, emerging evidence indicates that the stress response and molecular chaperones play an important role in stem cell oncogenesis $(10,30)$. In the present study, we found GRP78, a major stress-inducible ER chaperone which has been reported to play a crucial role in tumor therapeutic resistance $(9,31,32)$, was significantly increased in isolated SP cells. Based on the facts, both CSCs and GRP78 are closely associated with the resistance to cancer treatment. We therefore hypothesized that GRP78 may be involved in the radiation resistance of SP cells.

We thus directly knocked down the expression of GRP78 by transfecting with pSuper/GRP78 RNAi vector. The results showed that silencing GRP78 increased the effects of radiation, while increase of the expression of GRP78 elevated the resistance of SP to radiation. The present study demonstrated that the overexpression of GRP78 in SP cells mediates the resistance to radiation.

Collectively, the present study indicated that GRP78 plays an important role in maintaining the radiation resistance of SP cells. Based on our finding, targeting GRP78 may be a potential therapeutic target for eliminating breast CICs. Furthermore, combined with anti-GRP78 strategy, some cases of resistance to radiotherapy may be overcome.

\section{Acknowledgements}

The present study was supported by the National Natural Science Foundation of China (no. 30470846). The authors thank Professor Tianyan Zhou for the valuable input and suggestions.

\section{References}

1. Veronesi U, Boyle P, Goldhirsch A, Orecchia R and Viale G: Breast cancer. Lancet 365: 1727-1741, 2005.

2. Clarke M, Collins R, Darby S, et al: Effects of radiotherapy and of differences in the extent of surgery for early breast cancer on local recurrence and 15-year survival: an overview of the randomised trials. Lancet 366: 2087-2106, 2005.

3. Gebski V, Lagleva M, Keech A, Simes J and Langlands AO: Survival effects of postmastectomy adjuvant radiation therapy using biologically equivalent doses: a clinical perspective. J Natl Cancer Inst 98: 26-38, 2006.

4. Rosen JM and Jordan CT: The increasing complexity of the cancer stem cell paradigm. Science 324: 1670-1673, 2009.

5. Gupta PB, Chaffer CL and Weinberg RA: Cancer stem cells: mirage or reality? Nat Med 15: 1010-1012, 2009.

6. Phillips TM, McBride WH and Pajonk F: The response of $\mathrm{CD} 24(-/ \mathrm{low}) / \mathrm{CD} 44^{+}$breast cancer-initiating cells to radiation. J Natl Cancer Inst 98: 1777-1785, 2006.

7. Lagadec C, Vlashi E, Della Donna L, et al: Survival and self-renewing capacity of breast cancer initiating cells during fractionated radiation treatment. Breast Cancer Res 12: R13, 2010
8. Luo B and Lee AS: The critical roles of endoplasmic reticulum chaperones and unfolded protein response in tumorigenesis and anticancer therapies. Oncogene 32: 805-818, 2013.

9. Li J and Lee AS: Stress induction of GRP78/BiP and its role in cancer. Curr Mol Med 6: 45-54, 2006.

10. Mousa SA, Sudha T, Dyskin E, et al: Stress resistant human embryonic stem cells as a potential source for the identification of novel cancer stem cell markers. Cancer Lett 289: 208-216, 2010.

11. Luo S, Mao C, Lee B and Lee AS: GRP78/BiP is required for cell proliferation and protecting the inner cell mass from apoptosis during early mouse embryonic development. Mol Cell Biol 26: 5688-5697, 2006.

12. Bartkowiak K, Effenberger KE, Harder S, et al: Discovery of a novel unfolded protein response phenotype of cancer stem/ progenitor cells from the bone marrow of breast cancer patients. J Proteome Res 9: 3158-3168, 2010.

13. Wu MJ, Jan CI, Tsay YG, et al: Elimination of head and neck cancer initiating cells through targeting glucose regulated protein78 signaling. Mol Cancer 9: 283, 2010.

14. Goodell MA, Brose K, Paradis G, Conner AS and Mulligan RC: Isolation and functional properties of murine hematopoietic stem cells that are replicating in vivo. J Exp Med 183: 1797-1806, 1996.

15. Wright MH, Calcagno AM, Salcido CD, Carlson MD, Ambudkar SV and Varticovski L: Brcal breast tumors contain distinct $\mathrm{CD}_{4} 4^{+} / \mathrm{CD} 24^{-}$and $\mathrm{CD} 133^{+}$cells with cancer stem cell characteristics. Breast Cancer Res 10: R10, 2008.

16. Kondo T, Setoguchi T and Taga T: Persistence of a small subpopulation of cancer stem-like cells in the C6 glioma cell line. Proc Natl Acad Sci USA 101: 781-786, 2004.

17. Patrawala L, Calhoun T, Schneider-Broussard R, Zhou J, Claypool K and Tang DG: Side population is enriched in tumorigenic, stem-like cancer cells, whereas $\mathrm{ABCG} 2^{+}$and ABCG2- cancer cells are similarly tumorigenic. Cancer Res 65: 6207-6219, 2005.

18. Xia S, Zhao Y, Yu S and Zhang M: Activated PI3K/Akt/COX-2 pathway induces resistance to radiation in human cervical cancer HeLa cells. Cancer Biother Radiopharm 25: 317-323, 2010.

19. Al-Hajj M, Wicha MS, Benito-Hernandez A, Morrison SJ and Clarke MF: Prospective identification of tumorigenic breast cancer cells. Proc Natl Acad Sci USA 100: 3983-3988, 2003.

20. Pastrana E, Silva-Vargas V and Doetsch F: Eyes wide open: a critical review of sphere-formation as an assay for stem cells. Cell Stem Cell 8: 486-498, 2011.

21. Zhang P, Zhang Y, Mao L, Zhang Z and Chen W: Side population in oral squamous cell carcinoma possesses tumor stem cell phenotypes. Cancer Lett 277: 227-234, 2009.

22. Debeb BG, Xu W and Woodward WA: Radiation resistance of breast cancer stem cells: understanding the clinical framework. J Mammary Gland Biol Neoplasia 14: 11-17, 2009.

23. Stockler M, Wilcken NR, Ghersi D and Simes RJ: Systematic reviews of chemotherapy and endocrine therapy in metastatic breast cancer. Cancer Treat Rev 26: 151-168, 2000.

24. Al-Ejeh F, Smart CE, Morrison BJ, et al: Breast cancer stem cells: treatment resistance and therapeutic opportunities. Carcinogenesis 32: 650-658, 2011.

25. Fillmore CM and Kuperwasser C: Human breast cancer cell lines contain stem-like cells that self-renew, give rise to phenotypically diverse progeny and survive chemotherapy. Breast Cancer Res 10: R25, 2008.

26. Kakarala M and Wicha MS: Implications of the cancer stem-cell hypothesis for breast cancer prevention and therapy. J Clin Oncol 26: 2813-2820, 2008.

27. Ablett MP, Singh JK and Clarke RB: Stem cells in breast tumours: are they ready for the clinic? Eur J Cancer 48: 2104-2116, 2012.

28. Ho MM, Ng AV, Lam S and Hung JY: Side population in human lung cancer cell lines and tumors is enriched with stem-like cancer cells. Cancer Res 67: 4827-4833, 2007.

29. Chiba T, Kita K, Zheng YW, et al: Side population purified from hepatocellular carcinoma cells harbors cancer stem cell-like properties. Hepatology 44: 240-251, 2006.

30. Kang J, Shakya A and Tantin D: Stem cells, stress, metabolism and cancer: a drama in two Octs. Trends Biochem Sci 34: 491-499, 2009.

31. Dong D, Ko B, Baumeister P, et al: Vascular targeting and antiangiogenesis agents induce drug resistance effector GRP78 within the tumor microenvironment. Cancer Res 65: 5785-5791, 2005.

32. Fu Y,Li J and Lee AS: GRP78/BiP inhibits endoplasmic reticulum BIK and protects human breast cancer cells against estrogen starvation-induced apoptosis. Cancer Res 67: 3734-3740, 2007. 